\title{
Evaluation of Matrix-Assisted Laser Desorption Ionization Mass Spectrometry for Studying the sec-butyllithium and n-butyllithium Initiated Ring-Opening Polymerization of Hexamethylcyclotrisiloxane $\left(\mathrm{D}_{3}\right)$
}

\author{
Adam M. Hawkridge and Joseph A. Gardella, Jr. \\ Department of Chemistry, State University of New York at Buffalo, Buffalo, New York, USA
}

\begin{abstract}
Matrix assisted laser desorption ionization (MALDI) was used to study the organolithium initiated ring-opening polymerization of hexamethylcyclotrisiloxane $\left(\mathrm{D}_{3}\right)$ in a mixed solvent system. The mass spectral peak intensities were monitored to determine the effects of polymerization time, initiator concentration, and reaction temperature on the formation of the mono, di, and trisiloxanolate initiator species and the extent of chain redistribution. The three initiator species were formed by reacting n-butyllithium and sec-butyllithium with $D_{3}$ in nonpolar solvent. The mass spectral results showed that sec-butyllithium and n-butyllithium form different populations of initiator species under the same conditions and that the measured mass spectral peak intensities do not accurately represent the population of siloxanolate initiator species prior to propagation. The changes in peak intensities were attributed to chain redistribution. (J Am Soc Mass Spectrom 2003, 14, 95-101) (C) 2003 American Society for Mass Spectrometry
\end{abstract}

L inear polydimethylsiloxane (PDMS) is primarily synthesized by either the condensation of difunctional silanols or the cyclo-addition polymerizations of three (hexamethylcyclotrisiloxane, $\mathrm{D}_{3}$ ) or four (octamethylcyclotetrasiloxane, $\mathrm{D}_{4}$ ) membered siloxane rings $[1,2]$. The molecular weight and product purity in these systems are difficult to control because both methods are susceptible to side reactions that broaden the polydispersity $\left(\mathrm{M}_{\mathrm{w}} / \mathrm{M}_{\mathrm{n}}\right)$. The most common side reactions are chain redistribution and cyclization shown in Scheme 1. Chain redistribution occurs when a propagating chain reacts with another chain to remove a segment of polymer without terminating the reaction. Cyclization occurs when a propagating chain reacts with itself to form cyclic species $\left(\mathrm{D}_{3}, \mathrm{D}_{4}, \mathrm{D}_{5}\right.$, etc.). This type of side reaction is commonly referred to as chain backbiting. Lee et al. [3] first reported a new method for producing narrow molecular weight PDMS that was free of cyclic impurities. It was shown that n-butyllithium opened the $\mathrm{D}_{3}$ ring in nonpolar solvents to form lithium siloxanolate initiators but did not propagate

Published online January 6, 2003

Address reprint requests to Dr. J. A. Gardella, Department of Chemistry, State University of New York at Buffalo, 470 Natural Science Complex, Buffalo, NY 14260-3000, USA. E-mail: gardella@acsu.buffalo.edu without the addition of a small amount (1-3\%) of base promoting solvent (in this case these include polar aprotic solvents such as DMSO and THF). The control of the initiator basicity coupled with the high reactivity of the $\mathrm{D}_{3}$ monomer significantly reduced chain redistribution and eliminated the formation of cyclic impurities.

The reaction between organolithium initiators and $\mathrm{D}_{3}$ is not well understood due in large part to the unique behavior of the initiators and the lithium counterions in solution. Organolithium compounds associate in nonpolar solvents to form aggregated species of various degrees due to the electron deficient nature of the lithium atom [4]. N-butyllithium, for example, forms a hexameric aggregate [5] whereas sec-butyllithium forms a tetrameric aggregate [6] in hydrocarbon solvents. Studies involving the reaction of organolithium species with styrene $[7,8]$ and dienes $[8,9]$ have shown that the degree of association directly affects the reactivity. The less associated organolithium species are found to be generally more reactive. Upon reacting with $\mathrm{D}_{3}$, it has been proposed that the lithium counter ions associate to form lithium siloxanolate aggregates. The degree to which these proposed structures are associated and the mechanism that allows a $\mathrm{D}_{3}$ molecule to react with the aggregated lithium siloxanolate end is 
a

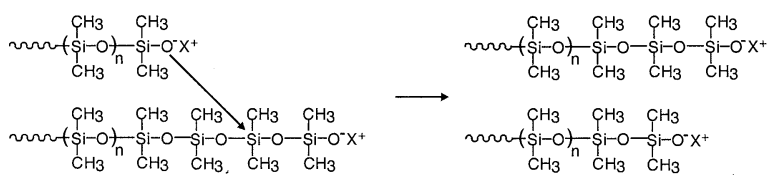

b

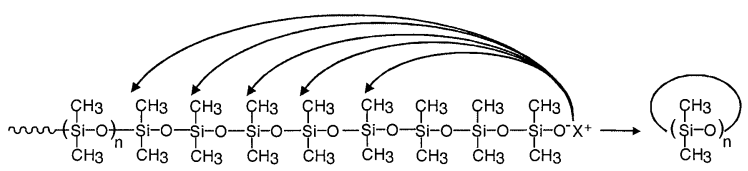

Scheme 1. Chain redistribution (a) and chain backbiting (b) mechanisms for anionically synthesized PDMS.

unclear. Thus, the formation and reaction kinetics of the lithium siloxanolate initiating species has been an active area of research for more than thirty years.

Frye et al. [10] studied the reactions between several organolithium reagents and a series of siloxane monomers in an attempt to identify the lithium siloxanolate initiator species. The gel permeation chromatography results showed that n-butyllithium reacted with $D_{3}$ to form three lithium siloxanolate species that are shown in Scheme 2. When the n-butyllithium and $D_{3}$ were mixed in a 3:1 ratio in hexane, the monosiloxanolate (n-1) species was found to form exclusively. Fessler et al. [11] determined the relative oligomerization rates of $\mathrm{n}-1$ towards $\mathrm{D}_{3}$ and $\mathrm{D}_{4}$ in various solvents. The study by Wilczek et al. [12] investigated the aggregation phe-

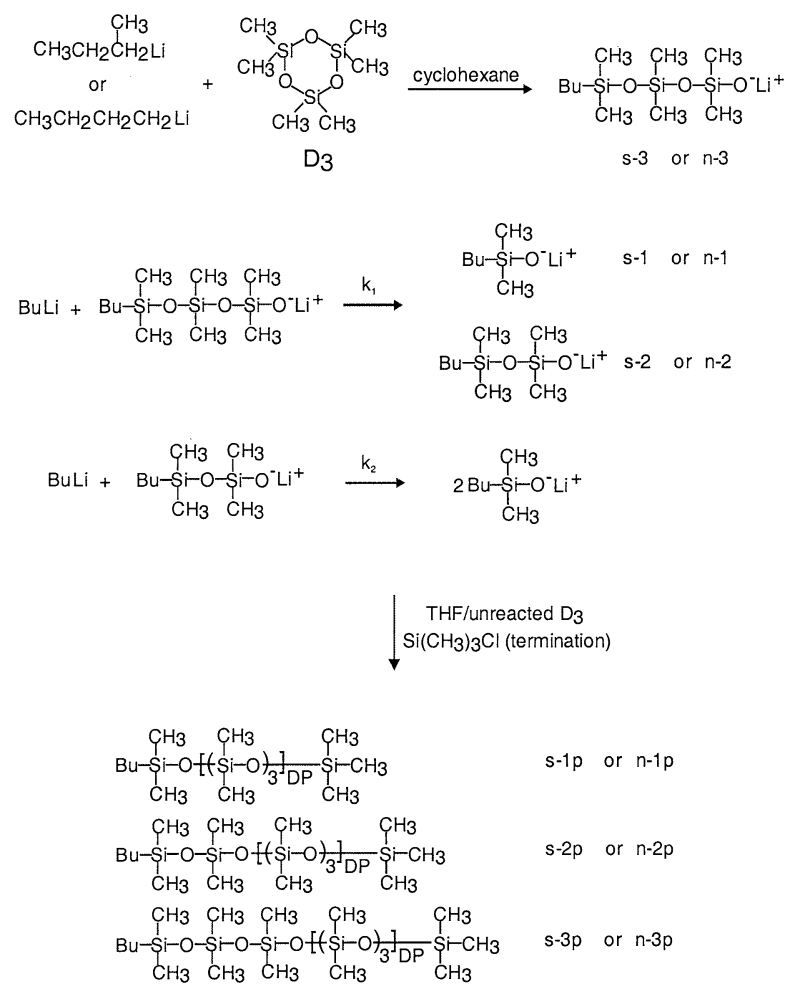

Scheme 2. Sec-butyllithium andd n-butyllithium initiated ringopening polymerization of hexamethylcyclotrisiloxane $\left(D_{3}\right)$. nomena associated with the organolithium/ $\mathrm{D}_{3}$ system and showed that the degree of aggregation dominated the kinetics of polymerization for a variety of temperatures.

A new approach for studying these systems was introduced by Hunt et al. [13, 14] where time of flight secondary ion mass spectrometry (TOF-SIMS) was used to determine the relative amounts of initiator species (Scheme 2: s-1, -2, and -3) from the sec-butyllithium initiated ring-opening polymerization of $\mathrm{D}_{3}$. Unlike the earlier studies that were restricted to analyzing low molecular weight oligomers, Hunt and coworkers analyzed the higher mass final polymer product. The resulting mass spectra showed a repeating pattern every three repeat units $(74 \mathrm{~m} / \mathrm{z})$ that correlated to the relative population of initiator species. Similar repeat patterns were observed by Yan et al. while analyzing a series of commercially obtained PDMS samples by TOF-SIMS, matrix assisted laser desorption ionization mass spectrometry (MALDI), and electrospray ionization mass spectrometry (ESI-MS) [15]. The mass spectral results for different molecular weight PDMS samples suggested that sec-butyllithium formed different amounts of siloxanolate initiator compared with the earlier findings on n-butyllithium initiated systems. Haddleton et al. [16] recently reported on MALDI results from n-butyllithium initiated $D_{3}$ polymerizations in THF. This study confirms that there are different populations of siloxanolate species and that they change as a function of reaction conditions. This change in peak intensities was attributed to side reactions such as chain redistribution and backbiting. Other studies have also used mass spectrometry to determine structural and mechanistic information on polymeric systems that would not be possible with other techniques [17-20].

This study addresses a number of fundamental issues in organolithium initiated polymerizations of $D_{3}$ by using MALDI mass spectrometry. The first goal of this study was to establish a quantitative way of representing the mass spectral peak populations in order to make empirical comparisons between spectra. This is essential for extracting kinetic information. Secondly, we wanted to determine whether the relative peak intensities at each degree of polymerization in the mass spectra were true representations of the siloxanolate initiator populations. The earlier mass spectral studies did not establish this [13-15]. The third issue addressed in this study was to evaluate the changes in peak intensities as a function of reaction conditions such as the polymerization time, initiator concentration, and the reaction temperature. Unlike Haddleton's work that looked at the effects of polymerization time for nbutyllithium initiated $\mathrm{D}_{3}$ in neat THF, we were interested in a mixed solvent system that allowed the initiators to form before polymerization started (i.e., before the base promoting THF was added to the cyclohexane, Scheme 2). The final objective was to 
directly compare the behavior of sec-butyllithium and n-butyllithium under identical reaction conditions.

\section{Experimental}

\section{Materials}

Hexamethylcyclotrisiloxane $\left(\mathrm{D}_{3}\right)$ (Gelest, Tullytown, PA, 98\%) was dried over calcium hydride for $24 \mathrm{~h}$ in an argon atmosphere. The cyclohexane and tetrahydrofuran was dried over sodium/benzephenone in an argon environment for at least three days prior to use. Secbutyllithium (Aldrich, Milwaukee, WI, 1.3 M in cyclohexane) and n-butyllithium (Aldrich, 1.6 M in cyclohexane) were used as received and titrated at least three times before each experiment using the method described by Gilman [21]. The diethylether (Mallinkrodt, Paris, KY) was dried over sodium/benzephenone in argon for at least three days and the dibromoethane (Aldrich) was dried over phoshorous pentoxide (Fisher, Pittsburgh, PA) for $12 \mathrm{~h}$ prior to use. The terminating species (trimethylchlorosilane, Gelest) was used as received. All glassware was dried overnight in an oven at $110{ }^{\circ} \mathrm{C}$. All of the dried reagents were transferred via a vacuum transfer line or gas-tight syringes.

\section{PDMS Synthesis (Time)}

Two separate $54 \mathrm{~mL}$ solutions of $0.6 \mathrm{M} \mathrm{D}_{3}$ /cyclohexane were prepared and stirred over a positive pressure of argon at $26^{\circ} \mathrm{C}$. A $1.60 \mathrm{~mL}\left(8.0 \times 10^{-4}\right.$ moles $)$ aliquot of a $0.50 \pm 0.04 \mathrm{M}$ sec-butyllithium/cyclohexane solution was added to one of the $\mathrm{D}_{3} /$ cyclohexane solutions and allowed to stir for $2 \mathrm{~h}$ over argon. A $0.38 \mathrm{~mL}\left(8.1 \times 10^{-4}\right.$ moles) aliquot of a $2.13 \pm 0.02 \mathrm{M}$ n-butyllithium/ cyclohexane solution was added to the second $\mathrm{D}_{3} /$ cyclohexane solutions and stirred for $2 \mathrm{~h}$ over argon. After two h of stirring, $6.0 \mathrm{~mL}$ of dry THF was added to both solutions over vigorous stirring. The polymerizations were carried out at $26^{\circ} \mathrm{C}$ over an argon atmosphere. $10.0 \mathrm{~mL}$ aliquots were removed from these two solutions via a gas-tight syringe at 3, 6, 12 and $24 \mathrm{~h}$ and transferred to separate argon charged flasks and stirred. After transferring each $10 \mathrm{~mL}$ aliquot to the argon charged flasks, $0.50 \mathrm{~mL}$ of trimethylchlorosilane was added to terminate the polymerization. Excess reagents were removed by vacuum and the resulting residue was immediately dissolved in methylene chloride. The organic solution was washed three times with distilled water. The methylene chloride was then removed by vacuum.

\section{PDMS Synthesis (Concentration)}

Two separate $30.0 \mathrm{~mL} 0.6 \mathrm{M} \mathrm{D}_{3}$ /cyclohexane solutions were prepared and stirred over a positive pressure of argon at $26^{\circ} \mathrm{C}$. For each initiator, three flasks were prepared with $9.0 \mathrm{~mL}$ aliquots of the $0.6 \mathrm{M}$ solution. For the sec-butyllithium; $0.45 \mathrm{~mL}\left(7.2 \times 10^{-4}\right.$ moles $), 0.30$
$\mathrm{mL}\left(4.8 \times 10^{-4}\right.$ moles $)$, and $0.15 \mathrm{~mL}\left(2.4 \times 10^{-4}\right.$ moles $)$ of a $1.61 \pm 0.04 \mathrm{M}$ sec-butyllithium/cyclohexane solution were added to each flask via a gas-tight syringe over vigorous stirring. For the n-butyllithium, $0.40 \mathrm{~mL}$ (7.5 $\times 10^{-4}$ moles), $0.27 \mathrm{~mL}\left(5.0 \times 10^{-4}\right.$ moles $)$, and 0.13 $\mathrm{mL}\left(2.5 \times 10^{-4}\right.$ moles $)$ of a $1.88 \pm 0.03 \mathrm{M}$ n-butyllithium/cyclohexane solution were added to each flask via a gas-tight syringe over vigorous stirring. After $2 \mathrm{~h}$ of stirring over argon, $1.0 \mathrm{~mL}$ of dry THF was added to each flask and the polymerizations were allowed to proceed for $48 \mathrm{~h}$. The polymerizations were carried out at $26^{\circ} \mathrm{C}$ over an argon atmosphere. At the end of $48 \mathrm{~h}$, the polymerizations were terminated with trimethylchlorosilane and purified by the aforementioned method.

\section{PDMS Synthesis (Temperature)}

Two separate $72.0 \mathrm{~mL} 0.6 \mathrm{M} \mathrm{D}_{3}$ /cyclohexane solutions were prepared and stirred over a positive pressure of argon at $26^{\circ} \mathrm{C}$. For each initiator, three flasks were prepared with $9.0 \mathrm{~mL}$ aliquots of the $0.6 \mathrm{M}$ solution. Each flask was immersed in temperature-controlled baths that were held at 0,30 , and $45^{\circ} \mathrm{C}$ and stirred for $20 \mathrm{~min}$. For the sec-butyllithium, $0.50 \mathrm{~mL}\left(1.4 \times 10^{-4}\right.$ moles) of a $0.29 \pm 0.01 \mathrm{M}$ sec-butyllithium/cyclohexane solution was added to each solution via a gas-tight syringe. For the n-butyllithium, $0.50 \mathrm{~mL}\left(1.4 \times 10^{-4}\right.$ moles) of a $0.29 \pm 0.02 \mathrm{M}$ n-butyllithium/cyclohexane solution was added to each flask via a gas-tight syringe. The solutions were stirred over argon for $2 \mathrm{~h}$ before adding $1.0 \mathrm{~mL}$ of dry THF to each flask. The polymerizations were terminated with trimethylchlorosilane after $12 \mathrm{~h}$. The final PDMS product was purified in the same way as described previously.

\section{Instrumentation and Sample Preparation}

The polymer samples were analyzed on a Perseptive Biosystems (Foster City, CA) Voyager D-7 matrix assisted laser desorption ionization mass spectrometer (MALDI-TOF) equipped with a $337 \mathrm{~nm} \mathrm{~N}_{2}$ laser and a time of flight analyzer. The instrument was operated in the positive ion linear mode with an accelerating voltage of $25 \mathrm{kV}$. The samples were prepared by depositing $2.0 \mu \mathrm{l}$ of a $30 \mathrm{mg} / \mathrm{mL}$ 2,5-dihydroxybenzoic acid (DHB)/THF solution onto a gold coated sample plate. The THF was allowed to evaporate prior to depositing $1.0 \mu \mathrm{l}$ of a 0.2 to $1.8 \mathrm{mg} / \mathrm{mL}$ PDMS/THF solution depending on the molecular weight of the polymer sample. The sample was introduced into the instrument after allowing the THF to evaporate for $10 \mathrm{~min}$.

\section{Results and Discussion}

The degree of polymerization (DP) is defined as the number of monomer units in a polymer chain. In the organolithium initiated ring-opening polymerization of $\mathrm{D}_{3}$, a degree of polymerization includes three siloxane 
units $\left(\left[\mathrm{Si}\left(\mathrm{CH}_{3}\right)_{2} \mathrm{O}\right]_{3}\right)$. This addition, however, occurs in the presence of three separate initiator species (Scheme 2) whose masses differ by $74 \mathrm{Da}$. Therefore, each three peak repeating pattern in a mass spectrum corresponds to a degree of polymerization. Scheme 2 shows the formation of the three initiator species prior to the addition of THF and their respective labels denoted as $s-1,-2$, or -3 and $n-1,-2$, or -3 . The label prefixes ("s" or " $n$ ") represent either sec-butyllithium or n-butyllithium. The polymer chains are labeled as $s-1 p,-2 p$, or $-3 p$ and $n-1 p,-2 p$, or $-3 p$. The mass spectral peaks corresponding to polymer chains with $\mathrm{Bu}\left[\mathrm{Si}\left(\mathrm{CH}_{3}\right)_{2} \mathrm{O}\right]_{1}\left\{\left[\mathrm{Si}\left(\mathrm{CH}_{3}\right)_{2} \mathrm{O}\right]_{3}\right\}_{\mathrm{DP}} \mathrm{Si}\left(\mathrm{CH}_{3}\right)_{3}$ are abbreviated as either s- or n-1p, chains with $\mathrm{Bu}\left[\mathrm{Si}\left(\mathrm{CH}_{3}\right)_{2} \mathrm{O}\right]_{2}\left\{\left[\mathrm{Si}\left(\mathrm{CH}_{3}\right)_{2} \mathrm{O}\right]_{3}\right\}_{D P} \mathrm{Si}\left(\mathrm{CH}_{3}\right)_{3}$ are abbreviated as s- or n-2p, and chains with $\mathrm{Bu}\left[\mathrm{Si}\left(\mathrm{CH}_{3}\right)_{2} \mathrm{O}\right]_{3}\left\{\left[\mathrm{Si}\left(\mathrm{CH}_{3}\right)_{2} \mathrm{O}\right]_{3}\right\}_{\mathrm{DP}} \mathrm{Si}\left(\mathrm{CH}_{3}\right)_{3}$ are abbreviated as s- or n-3p.

The relative intensities of the $s-$ or $n-1 p,-2 p$ and $-3 p$ polymer chains were determined by normalizing the intensities of the mass spectral peaks for each degree of polymerization.

$$
\begin{aligned}
& (\text { Normalized 1p })_{\mathrm{DP}}=1 \mathrm{p}_{\mathrm{DP}} /(1 \mathrm{p}+2 \mathrm{p}+3 \mathrm{p})_{\mathrm{DP}} \\
& (\text { Normalized 2p })_{\mathrm{DP}}=2 \mathrm{p}_{\mathrm{DP}} /(1 \mathrm{p}+2 \mathrm{p}+3 \mathrm{p})_{\mathrm{DP}} \\
& (\text { Normalized 3p })_{\mathrm{DP}}=3 \mathrm{p}_{\mathrm{DP}} /(1 \mathrm{p}+2 \mathrm{p}+3 \mathrm{p})_{\mathrm{DP}}
\end{aligned}
$$

The normalized intensities of the mass spectral peaks for each degree of polymerization were averaged across all degrees of polymerization in order to minimize mass discrimination effects that dominate in more polydisperse polymers $[22,23]$.

$$
\begin{aligned}
& \text { Average } 1 \mathrm{p}=\left[(\text { Normalized } 1 \mathrm{p})_{\mathrm{DP}}+(\text { Normalized }\right. \\
& \left.1 \mathrm{p})_{\mathrm{DP}+1}+\ldots+(\text { Normalized } 1 \mathrm{p})_{\mathrm{DP}+\mathrm{n}}\right] /(\mathrm{n}+1) \\
& \text { Average } 2 \mathrm{p}=\left[(\text { Normalized } 2 \mathrm{p})_{\mathrm{DP}}+(\text { Normalized }\right. \\
& \left.2 \mathrm{p})_{\mathrm{DP}+1}+\ldots+(\text { Normalized } 2 \mathrm{p})_{\mathrm{DP}+\mathrm{n}}\right] /(\mathrm{n}+1) \\
& \text { Average } 3 \mathrm{p}=\left[(\text { Normalized } 3 \mathrm{p})_{\mathrm{DP}}+(\text { Normalized }\right. \\
& \left.3 \mathrm{p})_{\mathrm{DP}+1}+\ldots+(\text { Normalized } 3 \mathrm{p})_{\mathrm{DP}+\mathrm{n}}\right] /(\mathrm{n}+1)
\end{aligned}
$$

These three values were then averaged with the other two mass spectral data and reported in this study. Figure 1 shows two representative spectra for secbutyllithium initiated PDMS (Figure 1a) and n-butyllithium initiated PDMS (Figure 1b). It is clear that there are different peak intensities for each degree of polymerization. Figure 2 shows the effect of polymerization time on the peak intensities for sec-butyllithium at a DP of 6 and for n-butyllithium at a DP of 9 . The polymerization time dependence on the mass spectral peak intensities was measured to determine if the intensity values were true representations of the siloxanolate initiator populations. The mass spectra of polymers initiated with sec-butyllithium showed significant populations of $\mathrm{s}-1 \mathrm{p}$ and $\mathrm{s}-2 \mathrm{p}$ peaks with smaller contributions from $s-3 p$ after $3 \mathrm{~h}$ of polymerization. As the polymerization continued, the $s-3 p$ peak intensity increased while the $s-1 p$ peak intensity decreased. The

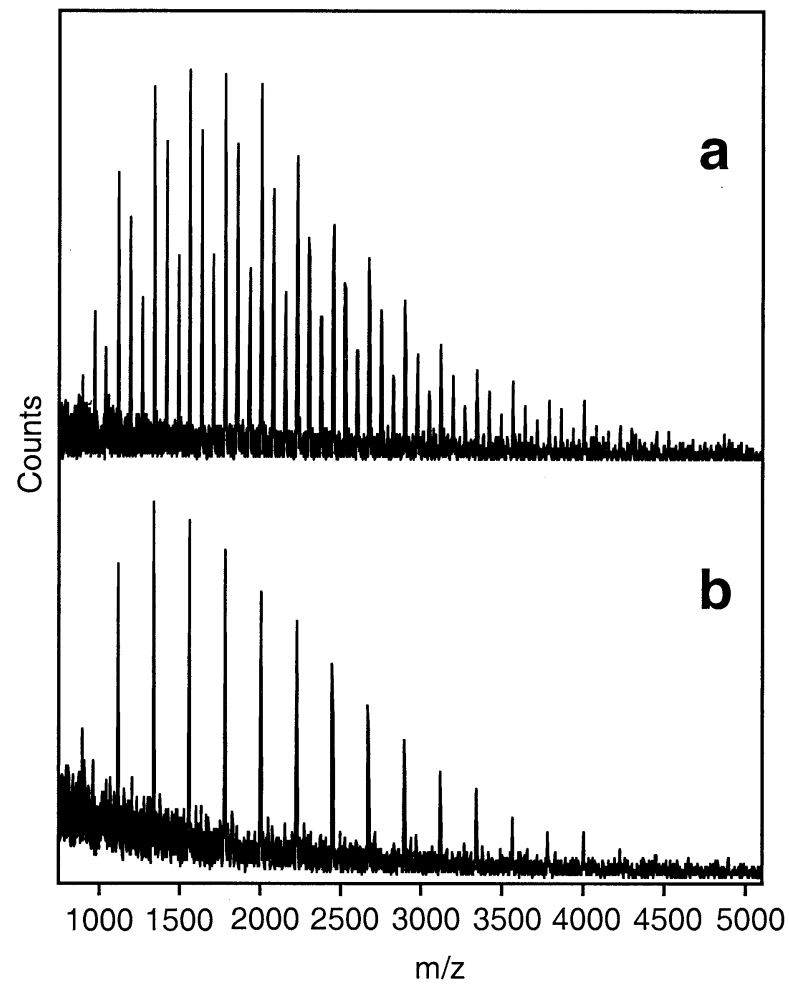

Figure 1. Positive ion spectra for sec-butyllithium (a) and nbutyllithium (b) initiated polymerizations of PDMS.

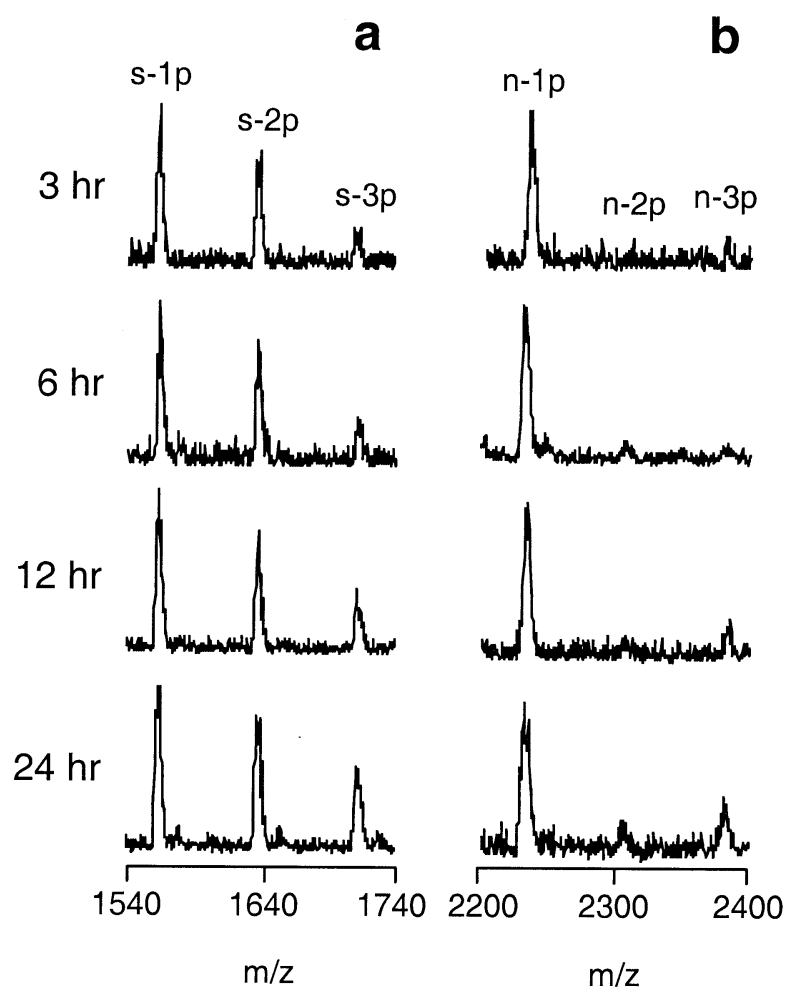

Figure 2. Positive ion spectra of one degree of polymerization (DP) for both sec- butyllithium (a), (DP = 6) and n-butyllithium (b), (DP = 9) initiated polymerizations after 3, 6, 12, and $24 \mathrm{~h}$ of polymerization. 
n-butyllithium analogues behaved similarly in that the $n-2 p$ and $n-3 p$ intensities increased at the expense of the $\mathrm{n}-1 \mathrm{p}$ peaks. These results established that the populations of the three polymer chain types do not accurately represent the initial populations of initiator species (e.g., s-1 $\neq \mathrm{s}-1 \mathrm{p})$.

The quantitative changes from the mass spectra of both the n-butyllithium and sec-butyllithium initiated PDMS are shown in Figure 3. Both initiator systems show statistically significant changes in the three types of polymer chains as the polymerization time increased. Previous studies on these systems in polar solvents found that approximately $90 \%$ of the $\mathrm{D}_{3}$ was converted to polymer after $3 \mathrm{~h}$ above room temperature $[2,24]$. After this time, less favorable side reactions become more prevalent as the reactive monomer concentration decreased. Based on these previous studies, we must assume that the conversion of $\mathrm{D}_{3}$ to polymer occurs very rapidly and that the changes in Figure 3 cannot be attributed to the initiation step. The changes in Figure 3 are attributed to the redistribution of siloxane units from growing polymer chains. We conclude that if chain redistribution were not occurring, the polymer chains would only grow by three siloxane repeat units (i.e., the opening of the $\mathrm{D}_{3}$ ring). However, it is clear from the mass spectral peak intensities after $3 \mathrm{~h}$ of polymerization that the $\mathrm{s}-1$ and $\mathrm{n}-1$ initiator species dominate the overall population early in the reaction. The exact extent of this dominance prior to propagation cannot be determined from the current data. However, it is clear from the data that the formation constants for the sec-butylsiloxanolate initiators are considerably different than for the n-butylsiloxanolate initiators. This difference is believed to be the result of steric hinderence. Efficient nucleophilic attack of the tri-siloxanolate by unreacted organolithium species (Scheme 2) favors the n-butyllithium [10] opposed to the more sterically hindered sec-butyllithium. The $\alpha$-substituted sec-butyllithium slows the rate of s-3 cleavage and facilitates more time for $\mathrm{D}_{3}$ ring-cleavage.

Figure 4 shows the quantitative changes in peak intensities as a function of initiator concentration. The sec-butyllithium systems showed changes in peak intensities whereas the n-butyllithium did not show any changes over the concentration range studied. Figure $4 \mathrm{a}$ shows that lower concentrations of sec-butyllithium produce more $s-3 p$ than at higher concentrations. This is attributed to the initial siloxanolate populations in solution prior to propagation. The lower amounts of sec-butyllithium allow more s-3 to survive whereas at higher concentrations there is more unreacted secbutyllithium in solution to attack the s-3 and s-2 to form mono-siloxanolate (s-1). A more extensive study with a larger concentration window may yield changes in the n-butyllithium initiated systems.

Temperature is commonly used to vary polymerization rates and as such is an important reaction condition to study. Figure 5 shows the changes in peak intensities as a function of reaction temperature. Both the initiator
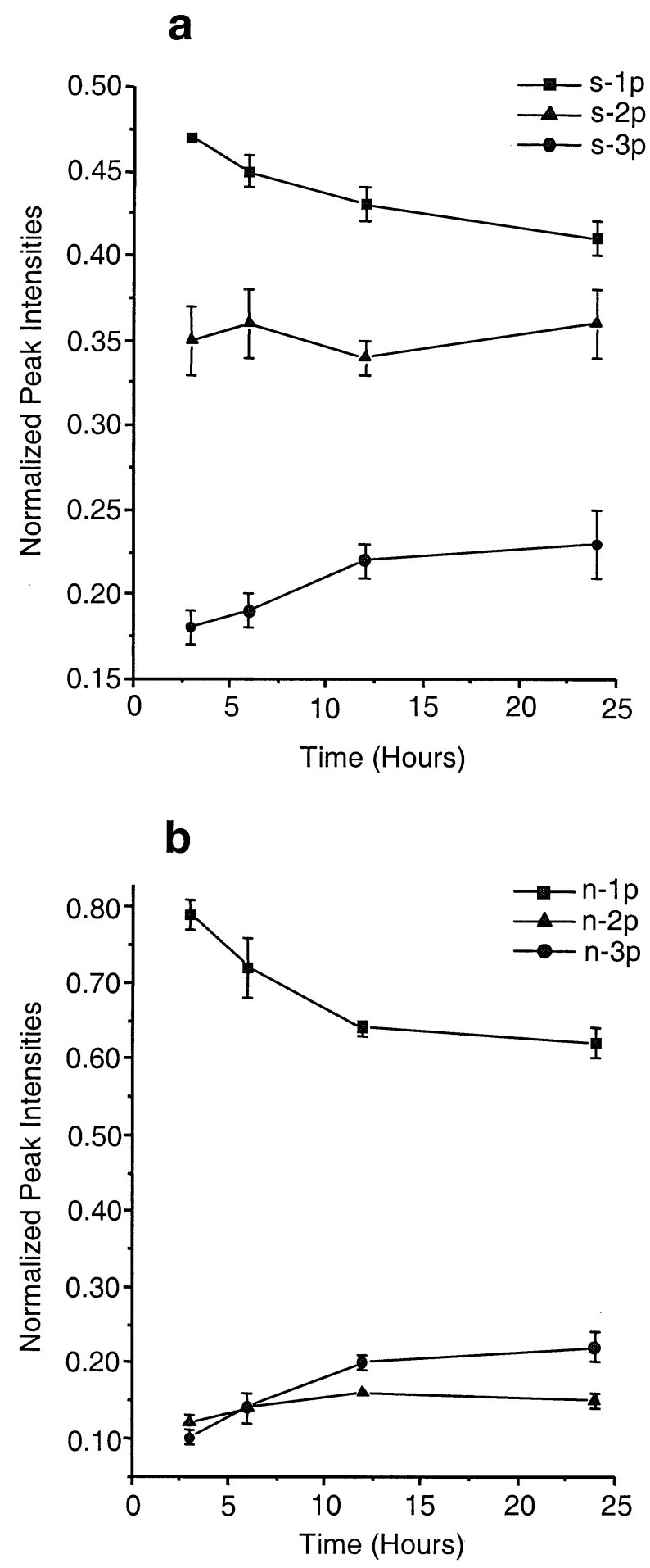

Figure 3. Average normalized mass spectral peak intensities plotted as a function of time: sec-butyllithium initiated polymerization (a) and n-butyllithium initiated polymerization (b).

and monomer concentrations were kept constant at each temperature. The sec-butyllithium and n-butyllithium show a sharp convergence between 30 and $45^{\circ} \mathrm{C}$. The $s-1 p$ and $n-1 p$ drastically decrease while $s-2 p, s-3 p$, $n-2 p$, and n-3p all increase. The sharp convergence at $45^{\circ} \mathrm{C}$ for both organolithium initiator species shows a 

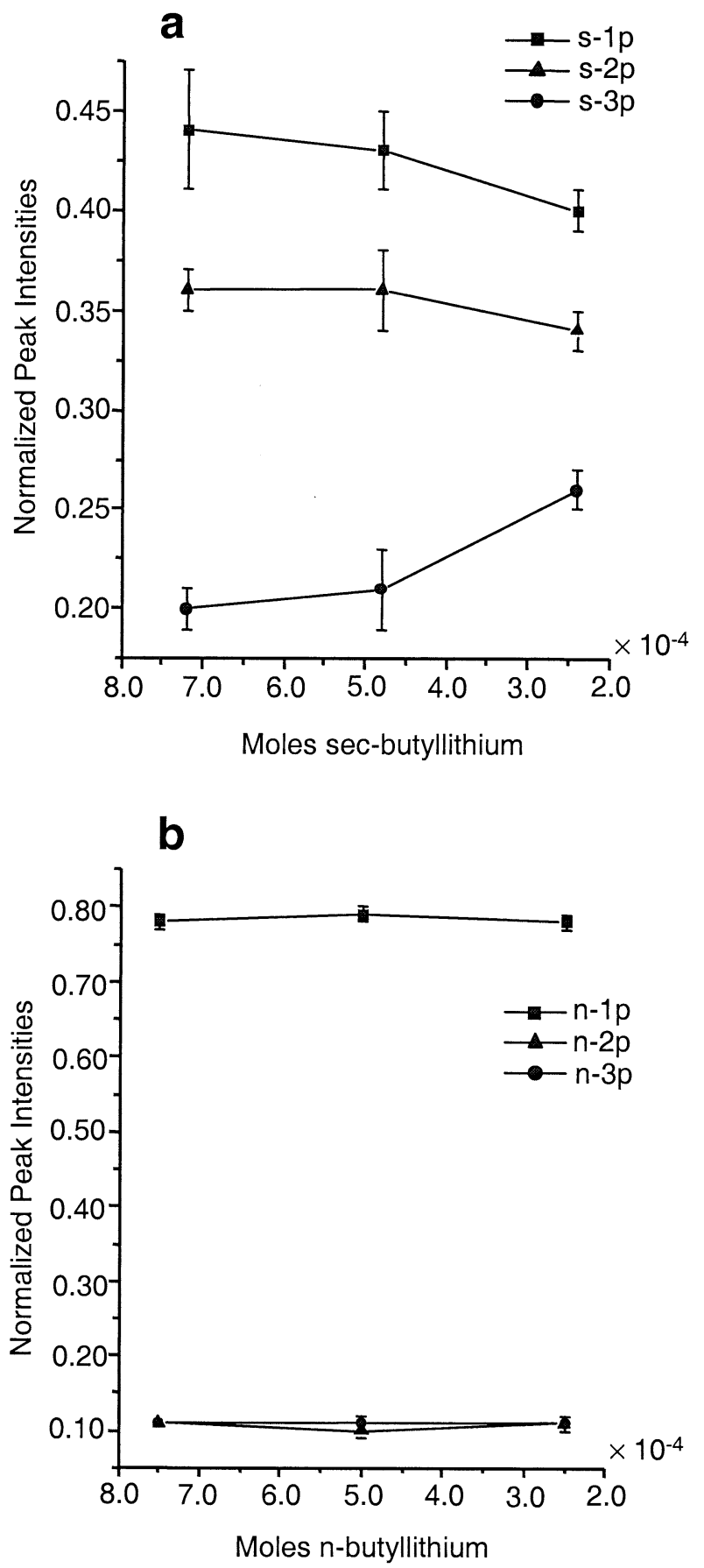

Figure 4. Average normalized mass spectral peak intensities plotted as a function of butyllithium concentration: sec-butyllithium initiated polymerization (a) and n-butyllithium initiated polymerization (b).

strong thermodynamic dependence for the rate of chain redistribution. The changes in peak intensities could be influenced by different initiator populations (i.e., $\mathrm{n}$ or $\mathrm{s}-1,2,3)$ but it is unlikely since an increase in temperature would favor more $\mathrm{n}-1$ or s-1.

Another aspect of Figures 3, 4, and 5 worth noting is that with the exception of Figure 5, the $s-2 p$ peak intensities are much higher relative to $s-3 p$ and $n-2 p$.
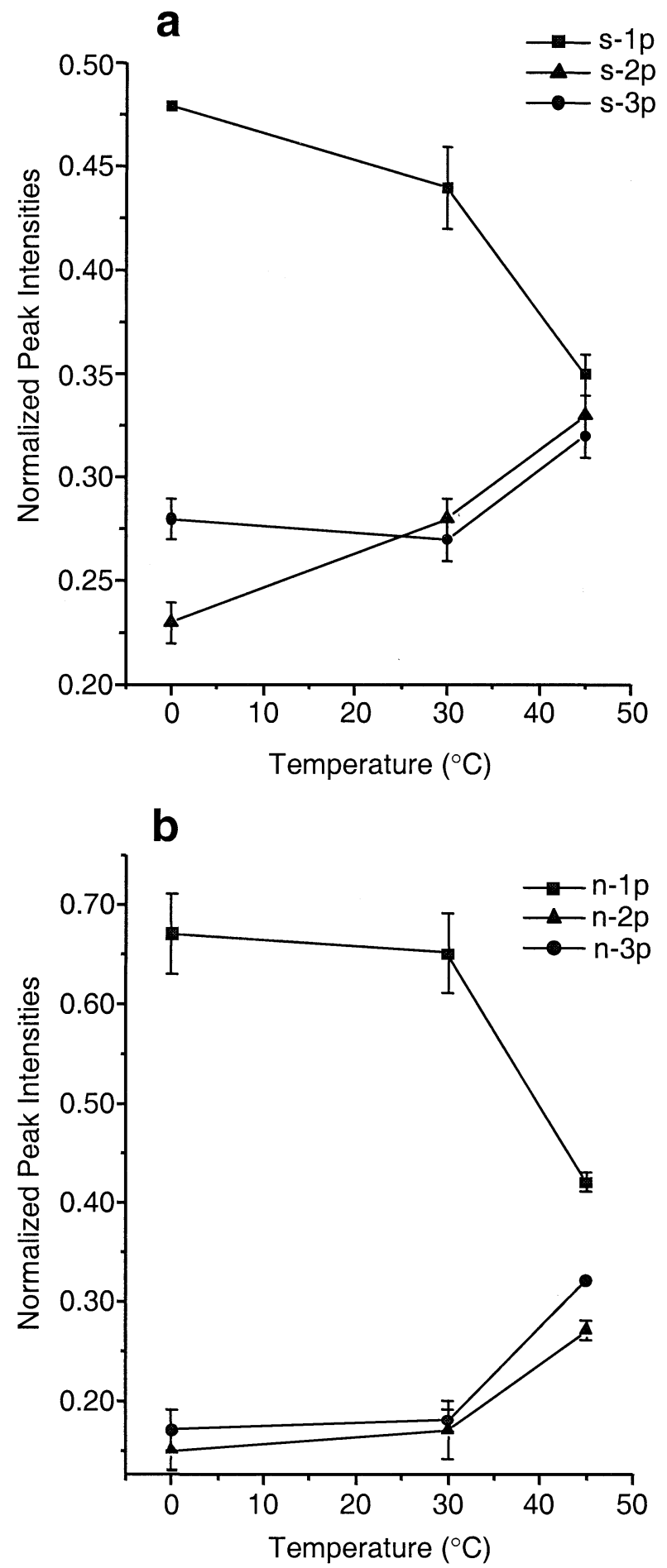

Figure 5. Average normalized mass spectral peak intensities plotted as a function of polymerization temperature: sec-butyllithium initiated polymerization (a) and n-butyllithium initiated polymerization (b).

For the n-butyllithium system, $\mathrm{k}_{1}$ and $\mathrm{k}_{2}$ (Scheme 2) are very fast [10]. This is corroborated by the data in Figures 3,4 , and 5 since both the $n-2 p$ and $n-3 p$ track parallel to one another and they are very small relative to $n-1 p$. However, the high population of $s-2 p$ chains suggests that $k_{2}$ for the sec-butyllithium system is 
limited relative to $k_{1}$. There must be some limiting factor that preferentially keeps a free sec-butyllithium molecule from attacking a s-2 over a s-3. This result cannot be rationalized by initiator sterics since reaction sites on s-2 are sterically equivalent to s-3. It may be possible that there is a s-2 aggregated structure that inhibits nucleophilic attack by an unreacted sec-butyllithium. If this is the case, a time dependent study on the formation of these three initiator species prior to polymerization would elucidate a kinetic dependence on initiator populations.

\section{Acknowledgments}

The authors gratefully acknowledge the financial support from NSF. The authors thank Dr. Timothy Koloski for his invaluable assistance in the synthesis of the polymers used in this study. They also thank Dr. Wenyan Yan, Dr. Dan Ammon, Dr. Pete Maziarz, Dr. Dan Hook, and Dr. George Grobe for their generous support and assistance on the MALDI at Bausch and Lomb, Rochester, New York. They thank Bausch and Lomb for the generousity of donating instrument time.

\section{References}

1. Saam, J. C. Formation of Linear Siloxane Polymers.In SiliconBased Polymer Science, A Comprehensive Resource. Advances in Chemistry 224; Zeigler, J. M.; Fearon, G. F. W., Eds.; American Chemical Society: Washington, D.C., 1990; pp. 71-90.

2. Wright, P. V. Cyclic Siloxanes. Ivin, K. J.; Saegusa, T., Eds. In Ring-opening Polymerization, Vol. II. Elsevier Applied Science Publishers: New York, 1984; pp. 1055-1133.

3. Lee, C. L.; Frye, C. L.; Johannson, O. K. Selective Polymerization of Reactive Cyclosiloxanes to Give Nonequilibrium Molecular Weight Distributions. Monodisperse Siloxane Polymers. Polym. Prepr. (Am. Chem. Soc., Div. Polym. Chem.) 1969, 10(2), 1361-1367.

4. Young, R. N.; Quirk, R. P.; Fetters, L. J. Anionic Polymerizations of Nonpolar Monomers Involving Lithium. In Anionic Polymerization. Springer-Verlag: New York, 1984; pp. 3-90.

5. Brown, T. L.; Ladd, J. A.; Newman, G. N. Interaction of Alkyllithium Compounds with Base. Complex Formation Between Ethyllithium and Lithium Ethoxide in Hydrocarbon Solvents. J. Organomet. Chem. 1965, 3, 1.

6. Bywater, S.; Worsfold, D. J. Alkyllithium Anionic Polymerization Initiators in Hydrocarbon Solvents. J. Organomet. Chem. 1967, 10, 1-6.

7. Hsieh, H. L. Kinetics of Polymerization of Butadiene, Isoprene, and Styrene with Alkyllithiums I. Rate of Polymerization. J. Polym. Sci. 1965, A-3, 153-161.

8. Hsieh, H. L. Kinetics of Polymerization of Butadiene, Isoprene, and Styrene with Alkyllithiums II. Rate of Initiation. J. Polym. Sci. 1965, A-3, 163-172.

9. Selman, C. M.; Hsieh, H. L. Effect of Aggregation Size on Alkyllithium Initiated Polymerizations. Polym. Lett. 1971, 9, 219.
10. Frye, C. L.; Salinger, R. M.; Fearon, F. W. G.; Klosowski, J. M.; DeYoung, T. Reactions of Organolithium Reagents with Siloxane Substrates. J. Org. Chem. 1970, 35, 1308-1314.

11. Fessler, W. A.; Juliano, P. C. Reactivity of Solvated Lithium n-Butyldimethylsilanolate with Organosiloxane Substrates. Ind. Eng. Chem. Prod. Dev. 1972, 11(4), 407-410.

12. Wilczek, L.; Kennedy, J. P. Aggregation in the Anionic Polymerization of Hexamethylcyclotrisiloxane with Lithium Counterion. Polym. J. 1987, 19(5), 531-538.

13. Hunt, M. O.; Belu, A. M.; Linton, R. W.; DeSimone, J. M. Elucidation of Initiating Species of Anionic Ring-Opening Polymerization of $\mathrm{D}_{3}$ by Time-of-Flight Secondary Ion Mass Spectrometry. Polym. Prepr. (Am. Chem. Soc. Div. Polym. Chem.) 1993, 34(2), 530-531.

14. Belu, A. M.; DeSimone, J. M.; Hunt, M. O.; Sheares, V. V.; Linton, R. W. Evaluation of Polymerization Mechanisms Using Time-of-Flight Secondary Ion Mass Spectrometry. Proceedings of the 9th SIMS Conference; Yokohama, Japan, November 7-12, 1993; pp 776-779.

15. Yan, W.; Ammon, D. M., Jr.; Gardella, J. A., Jr.; Maziarz, P. E., III; Hawkridge, A. M.; Grobe, G. L., III; Wood, T. W. Quantitative Mass Spectrometry of Technical Polymers: A Comparison of Several Ionization Methods. Eur. Mass Spectrom. 1998, 4, $467-474$.

16. Haddleton, D. M.; Bon, S. A. F.; Robinson, K. L.; Emery, N. J.; Moss, I. Matrix-Assisted Laser Desorption Ionization Time-ofFlight Mass Spectrometry of Polydimethylsiloxanes Prepared via Anionic Ring-Opening Polymerization. Macromol. Chem. Phys. 2000, 201, 694-698.

17. Nielen, M. W. F. MALDI Time-of-Flight Mass Spectrometry of Synthetic Polymers. Mass Spectrom. Rev. 1999, 18, 309-344.

18. Belu, A. M.; DeSimone, J. M.; Linton, R. W.; Lange, G. W.; Friedman, R. M. Evaluation of Matrix-Assisted Laser Desorption Ionization Mass Spectrometry for Polymer Characterization. J. Am. Soc. Mass Spectrom. 1996, 7, 11-24.

19. Sato, H.; Ohtani, H.; Tsuge, S.; Hayahshi, N.; Katoh, K.; Masuda, E.; Ohnishi, K. Structural Characterization of Polyoxymethylenes by Matrix-Assisted Laser Desorption/ Ionization Mass Spectrometry. Rapid Commun. Mass Spectrom. 2001, 15, 82-88.

20. Roberts, E. G.; Heuts, J. P. A.; Davis, T. P. Direct Observation of Cobalt-Carbon Bond Formation in the Catalytic Chain Transfer Polymerization of Methyl Acrylate Using MatrixAssisted Laser Desorption Ionization Time-of-Flight Mass Spectrometry. Macromolecules 2000, 33, 7765-7768.

21. Gilman, H.; Cartledge, F. K. The Analysis of Organolithium Compounds. J. Organomet. Chem. 1964, 2, 447-454.

22. Schriemer, D. C.; Liang, L. Mass Discrimination in the Analysis of Polydisperse Polymers by MALDI Time-of-Flight Mass Spectrometry. 1. Sample Preparation and Desporption/Ionization Issues. Anal. Chem. 1997, 69, 4169-4175.

23. Schriemer, D. C.; Liang, L. Mass Discrimination in the Analysis of Polydisperse Polymers by MALDI Time-of-Flight Mass Spectrometry. 2. Instrumental Issues. Anal. Chem. 1997, 69, $4176-4183$.

24. Holle, H. J.; Lehnen, B. R. Preparation and Characterization of Polydimethylsiloxanes with Narrow Molecular Weight Distribution. Europ. Polym. J. 1975, 11, 663-667. 